\title{
Hydrodynamische Modellierung von Oberflächengewässern
}

Zusammenfassung: In diesem Beitrag werden die hydrodynamischen Modellen zugrunde liegenden Modellansätze, die für die Erstellung solcher Modelle erforderlichen Datengrundlagen sowie die einzelnen Schritte im Zuge der hydraulischen Berechnung selbst überblicksartig dargestellt. In einem kurzen Ausblick werden zuletzt mögliche zukünftige Entwicklungen erwähnt.

Hydrodynamic modelling of surface water flow

Summary: This paper gives an overview of the approaches to hydrodynamic modelling of surface water flow, the required data base as well as the individual steps of the hydraulic calculations. Possible future developments are also discussed.

\section{Einleitung}

Die Berechnung von Abflüssen mittels hydrodynamischer Modelle ist eine in der Praxis unverzichtbare Methode zur Bestimmung von bei Hochwässern von Überflutung bedrohten Bereichen und zur Beurteilung des damit verbundenen Risikos. Zudem sind derartige Berechnungen die wesentlichen Hilfsmittel zur Konzeption und Optimierung neuer und zur Überprüfung bereits bestehender Wasserbauten, zur Beurteilung deren Auswirkungen auf das Umland und der Konsequenzen bei deren Versagen. Nicht zuletzt bilden die Ergebnisse solcher Berechnungen Grundlagen für zahlreiche andere Disziplinen wie Ökologie, Raum- und Verkehrsplanung, Energie- und Versicherungswirtschaft, etc.

\section{Modellansätze}

Ziel numerischer hydrodynamischer $\mathrm{Ab}$ flussmodellierungen ist die möglichst genaue Nachbildung von in der Natur auftretenden Strömungsverhältnissen, welche auf den physikalischen Masse-, Impulsund Energieerhaltungsgleichungen beru-

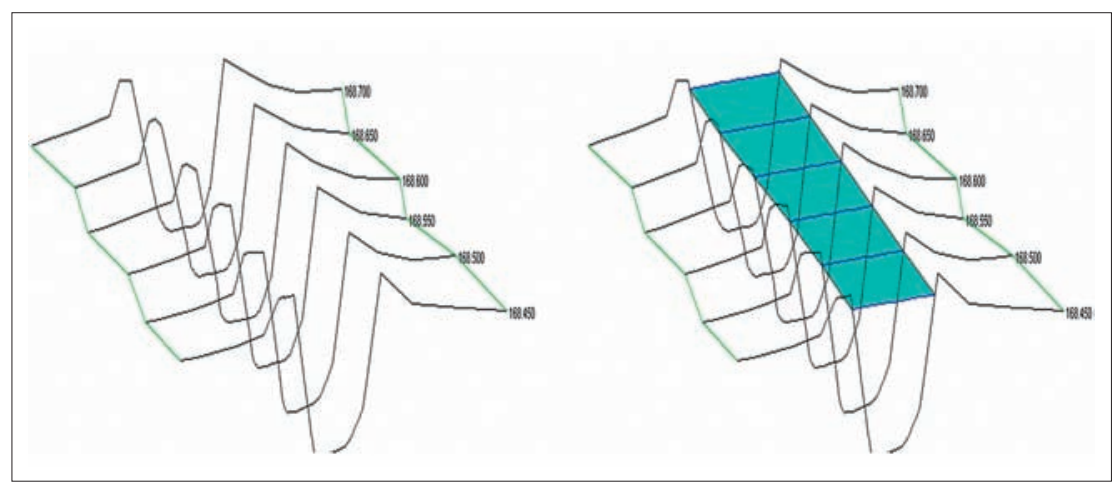

Abb. 1: 1d-Geometrie und 1d-Berechnungsergebnis.

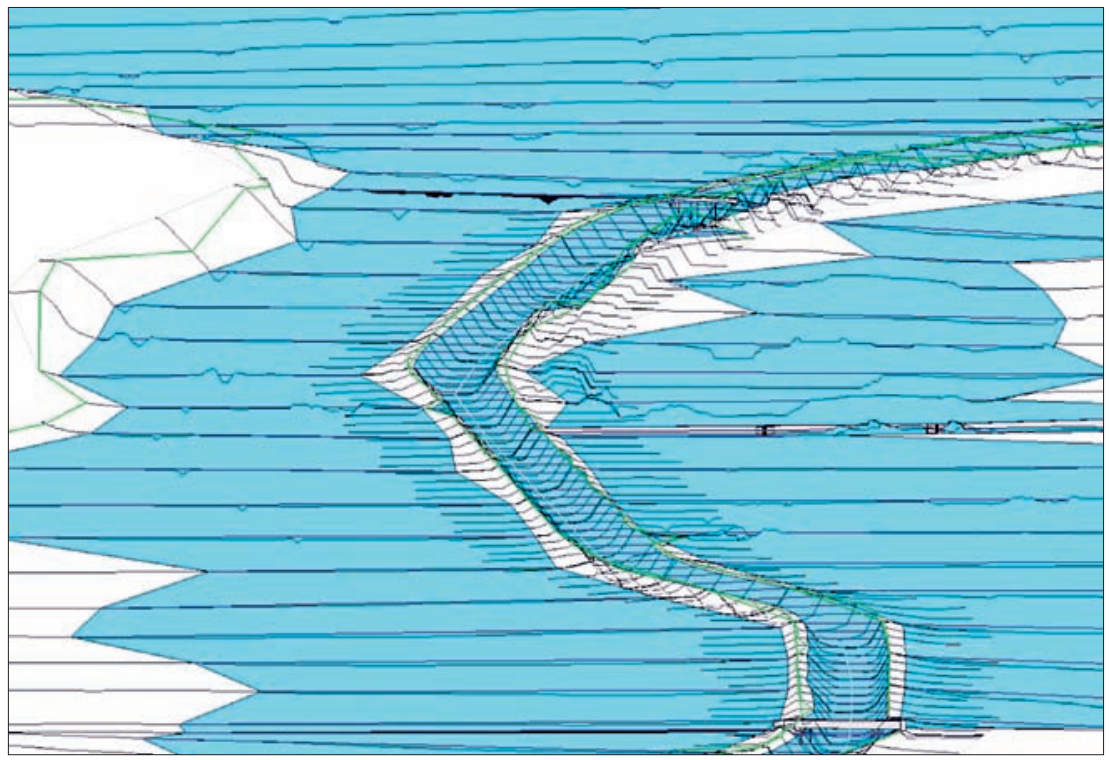

Abb. 2: 1d-Berechnungsergebnis komplexer Strömungsverhältnisse.

hen. Formuliert wurden diese Gleichungen bereits im 19. Jhdt von Claude Navier und George Stokes in einem nichtlinearen, partiellen Differenzialgleichungssystem 2. Ordnung, welches eine Flüssigkeitsströmung im Raum exakt beschreibt. Die Lösung dieses Gleichungssystems ist jedoch derzeit noch nicht mit vertretbarem Zeit- und Rechenaufwand für die in der Ingenieurpraxis auftretenden Fragestellungen möglich, da die dabei durchzuführenden Berechnungen kleinster Turbulenzen ein extrem feines Berechnungsgitter erfordern. Osborne Reynolds vereinfachte das Navier-Stokes'sche Gleichungssystem dahingehend, dass durch den Ansatz von Turbulenzmodellen die numerische Lösung großräumigerer Strömungsprobleme möglich wurde. Auch hierzu ist der zu untersuchende Raum zu diskretisieren, also in finite Recheneinheiten zu unterteilen. Sind für die ausreichende Beschreibung eines zu untersuchenden Strömungsproblems nicht alle drei Richtungen der Strömung erforderlich, so kann die Dimensionalität des Problems reduziert werden. Weiters können die Navier-Stokes'schen bzw. Reynolds-Gleichungen unter der An- 


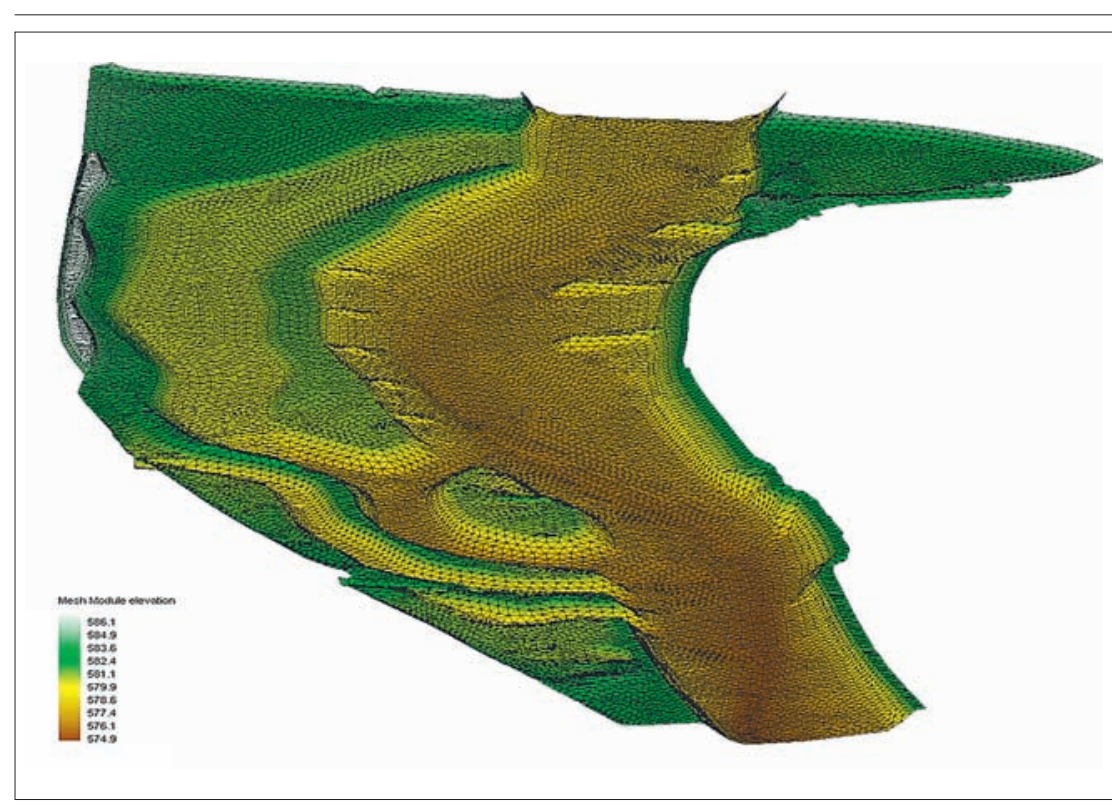

Abb. 3: 1d-Berechnungsergebnis komplexer Strömungsverhältnisse.

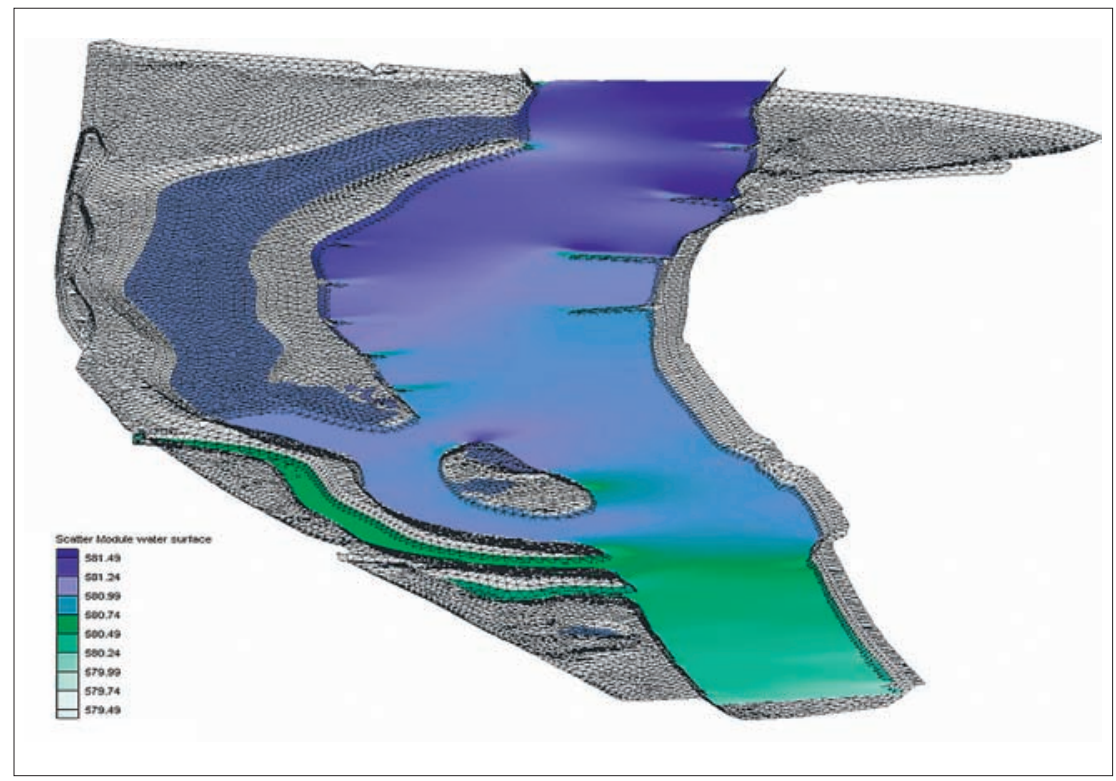

Abb. 4: 2d-Berechnungsergebnis - Wasserspiegellagen.

nahme, dass die flächige Ausdehnung eines Strömungsproblems deutlich größer als dessen Tiefe ist, dass hydrostatische Druckverteilung herrscht und dass die Geschwindigkeitskomponenten in vertikale Richtung vernachlässigbar sind $\mathrm{zu}$ den Saint-Venant'schen oder Flachwassergleichungen vereinfacht werden. Sie bilden die Grundlage für die in der Praxis gebräuchlichen 1d- und 2d-Modelle.

\subsection{1d-Modelle}

Bei 1d-Modellen wird neben den drei erwähnten Vereinfachungen zusätzlich angenommen, dass zur hinreichenden Be- lere Fließgeschwindigkeit sowie die mittlere Fließtiefe bzw. Lage des Wasserspiegels im Querprofil vor (Abb. 1).

Sofern die zugrundeliegenden Vereinfachungen dies zulassen, können durch eine entsprechende Gliederung des Abflussraumes durchaus auch komplexe Strömungsverhältnisse eindimensional berechnet werden (Abb. 2).

\subsection{2d-Modelle}

Bei 2d-Modellen werden zusätzlich zu den drei vereinfachenden Grundannahmen der Flachwassergleichungen die $\mathrm{Ge}$ schwindigkeitskomponenten in $\mathrm{x}$ - und $\mathrm{y}$ Richtung berücksichtigt und die Fließgeschwindigkeit lediglich über die Fließtiefe gemittelt. Die Lösung kann dann mittels der 2d-Flachwassergleichungen gefunden werden. Bei der Diskretisierung eines derart zu untersuchenden Abflussraumes wird dieser mit einem für die Lösung der Differenzialgleichungen passenden Netz aus Elementen und Knoten überzogen (Abb. 3). Die nach der Lösung vorliegenden Ergebnisse sind dann die Fließtiefe bzw. die Lage des Wasserspiegels sowie die Fließgeschwindigkeit und Fließrichtung an jedem Knoten ( $A b b .4$ ). Als dabei eingesetzte Lösungsverfahren kommen Methoden der Finiten Elemente, Methoden der Finiten Differenzen solche der Finiten Volumen in Frage.

\section{Erforderliche Datengrundlagen}

\subsection{Topografie}

Ein ausreichend hoch aufgelöstes Geländemodell des Abflussraumes stellt eine ganz wesentliche Grundlage für die hydrodynamische Berechnung von Oberflächengewässern dar. Basis dafür sind heute in der Regel bei Laserscannerbefliegungen (Airborne Laser Scanning, ALS) gewonnene Daten nach Durchführung der entsprechenden Prozessierung wie Georeferenzierung und Filterung ( $A b b .5)$. $\mathrm{Zu}$ ergänzen sind diese Laserscandaten durch Informationen über die Lage der Gewässersohle sowie die Geometrien abflussrelevanter Objekte wie Wehre, Durchlässe oder Brückenkonstruktionen. Diese sind mit Laserscanning in der Regel nicht erfassbar und entstammen üblicherweise konventionellen terrestrischen Vermessungen (Abb. 6 und 7).

Man muss sich bei der Verwendung topografischer Daten stets vor Augen halten, dass ein so erarbeitetes Geländemodell nur eine Momentaufnahme der Wirk- 
lichkeit abbildet. Infolge anthropogener Eingriffe oder zwischenzeitlich eingetretener Naturereignisse können Geländedaten an Aktualität verlieren, die Erfassung eines Flussbettes kann an oft besonders sensiblen Stellen innerhalb kurzer Zeit infolge Geschiebetrieb, Baggerungen etc. überholt sein.

Nicht unerwähnt soll auch bleiben, dass allfällig erforderliche terrestrische Messkampagnen heute häufig den größten Kostenfaktor im Rahmen von Fließgewässermodellierungen darstellen.

\subsection{Flächennutzung}

Sie ist die Grundlage für die in jedem Modell anzusetzende Rauigkeit zur Beschreibung der Widerstände beim Fließen über einen bestimmten Untergrund. Es ist leicht nachvollziehbar, dass ein Fließen über eine Asphaltfläche, eine Wiese oder durch einen dichten Auwald jeweils unterschiedlich erfolgt.

Beachtet werden muss dabei die jahreszeitliche Variabilität der Rauigkeiten. Speziell auf landwirtschaftlich genutzten Flächen können sich bei der Berechnung deutliche Unterschiede ergeben, je nach dem, ob Rauigkeiten entsprechend einem Acker zum Zeitpunkt der Aussaat oder einer Maiskultur in Vollreife angesetzt werden.

Grundlagen für eine Zuordnung von Rauigkeitswerten können aus den meist bereits digital vorliegenden Flächennutzungskarten abgeleitet werden. Weitere Quellen sind Luftbilder und, derzeit häufig als aktuellste Quelle beispielsweise zur Identifizierung sich ausbreitender Waldflächen oder Siedlungsgebiete, die Schummerung des digitalen Oberflächenmodells des Laserscans (Abb. 8). Überprüfungen vor Ort korrigieren und ergänzen die Zuordnung von Flächennutzungen bzw. Rauigkeiten.

\subsection{Hydrologie}

In den letzten Jahren ist die Hydrologie in verstärktem Ausmaß Gegenstand wissenschaftlicher Bearbeitungen. Als Resultat liegen heute beispielsweise für nahezu alle Oberflächengewässer Österreichs die Hochwasserabflussdaten des Projektes HORA vor. HORA steht für HochwasserRisikoflächen Austria und wurde nach den Verheerungen des Hochwassers 2002 als Gemeinschaftsprojekt vom Bundesministerium für Land- und Forstwirtschaft, Umwelt und Wasserwirtschaft und der Versicherungswirtschaft ins Leben gerufen. Die

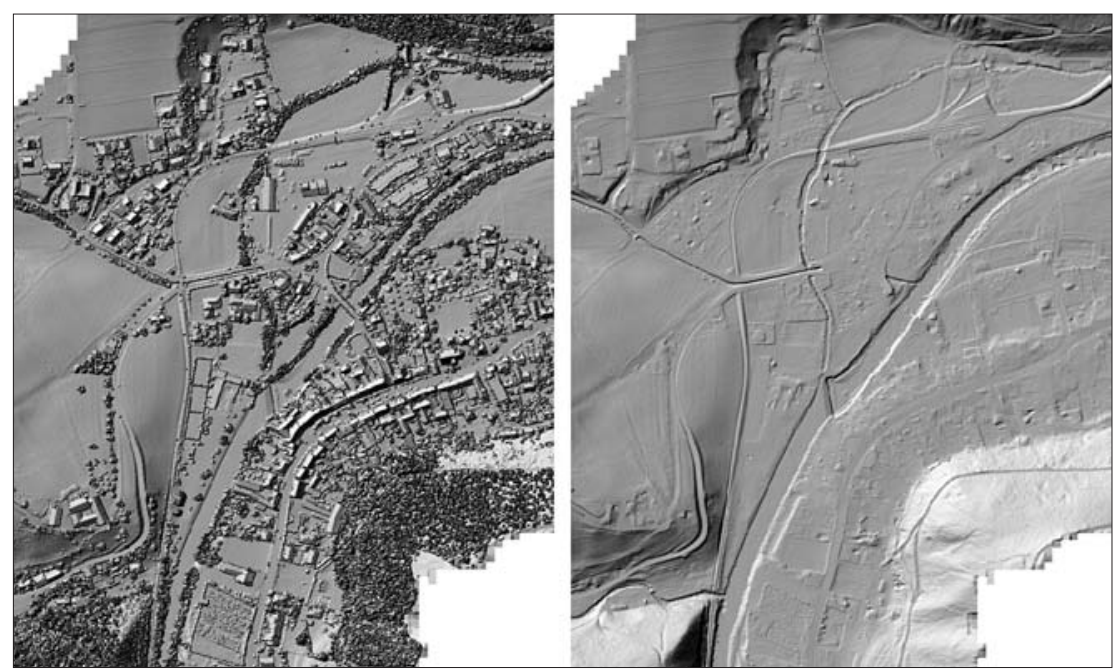

Abb. 5: ALS - Daten vor (Oberflächenmodell, links) und nach Filterung (Geländemodell, rechts).
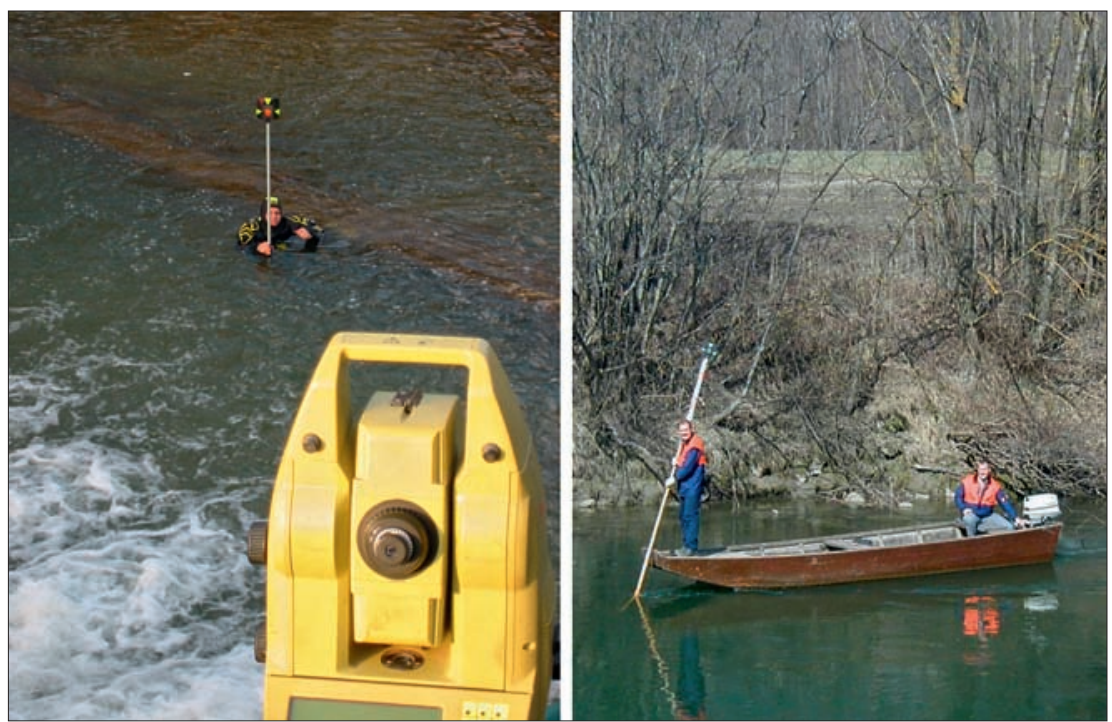

Abb. 6: Terrestrische Ergänzungsmessungen (Foto links: Vermessung Schubert, St. Pölten).

im Rahmen dieses Projektes von der TU Wien durchgeführten hydrologischen Bearbeitungen von über 10.000 Gewässerknoten basieren auf aktuellen wissenschaftlichen Erkenntnissen und Methoden. Als weitere wichtige Quelle sind die Hydrographischen Dienste der Länder und des Bundes zu nennen, welche ein mit über 950 Pegelstationen international vergleichsweise dichtes Messnetz betreuen. Für Gebiete ohne ausreichende hydrographische Datenbasis ist oft eine Herleitung hydrologischer Kenngrößen aus vergleichbaren benachbarten Einzugsgebieten zweckmäßig. Es kann aber auch erforderlich sein, die entsprechenden hydrologischen Bemessungsdaten anhand von oftmals aufwändig zu erstellenden Niederschlag-Abflussmodellen festzulegen. Ziel ist die Erarbeitung eines ausreichend detaillierten hydrologischen Längenschnittes, welcher die Ab- flussentwicklung im Längsverlauf unter BerücksichtigungderZwischeneinzugsgebiete und der relevanten Zubringer abbildet (Abb. 9).

Zumeist ist heute die Berechnung von Auswirkungen stationärer Abflüsse gefordert. Sind instationäre Vorgänge von Interesse, so sind zusätzlich abgelaufene Hochwasserwellen zu erheben und hinsichtlich Form und Fracht zu analysieren Nach verschiedenen Verfahren lassen sich so für Ereignisse bestimmter Scheitelwerte bzw. Jährlichkeiten oder bestimmter Fracht synthetische Hochwasserwellen generieren.

\subsection{Vorschriften}

Hierunter fallen im Hochwasserfall einzuhaltende Maßnahmen wie beispielsweise Wehrbetriebsordnungen, das Errichten 


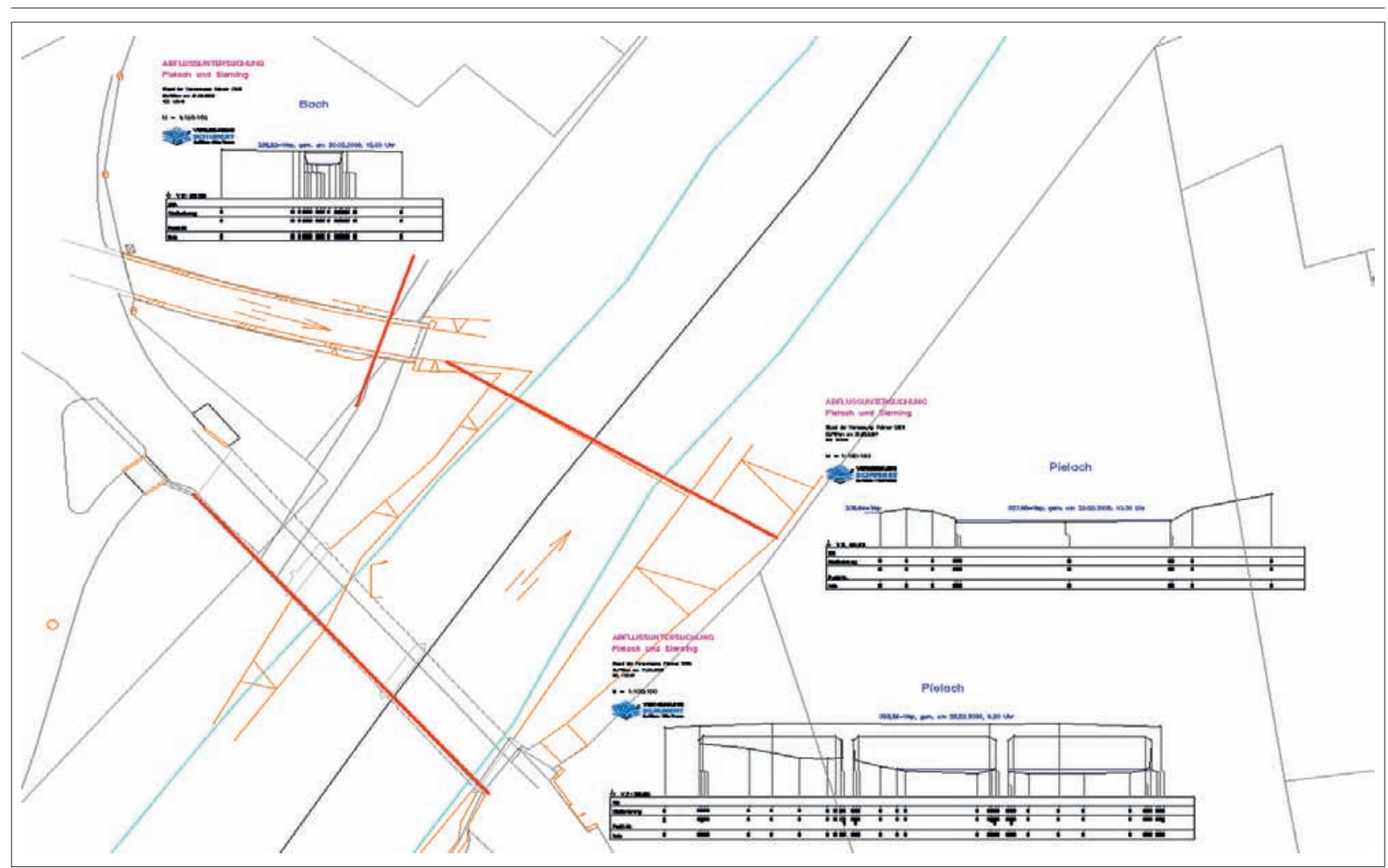

Abb. 7: Terrestrische Ergänzungsmessungen - Objektaufnahmen.

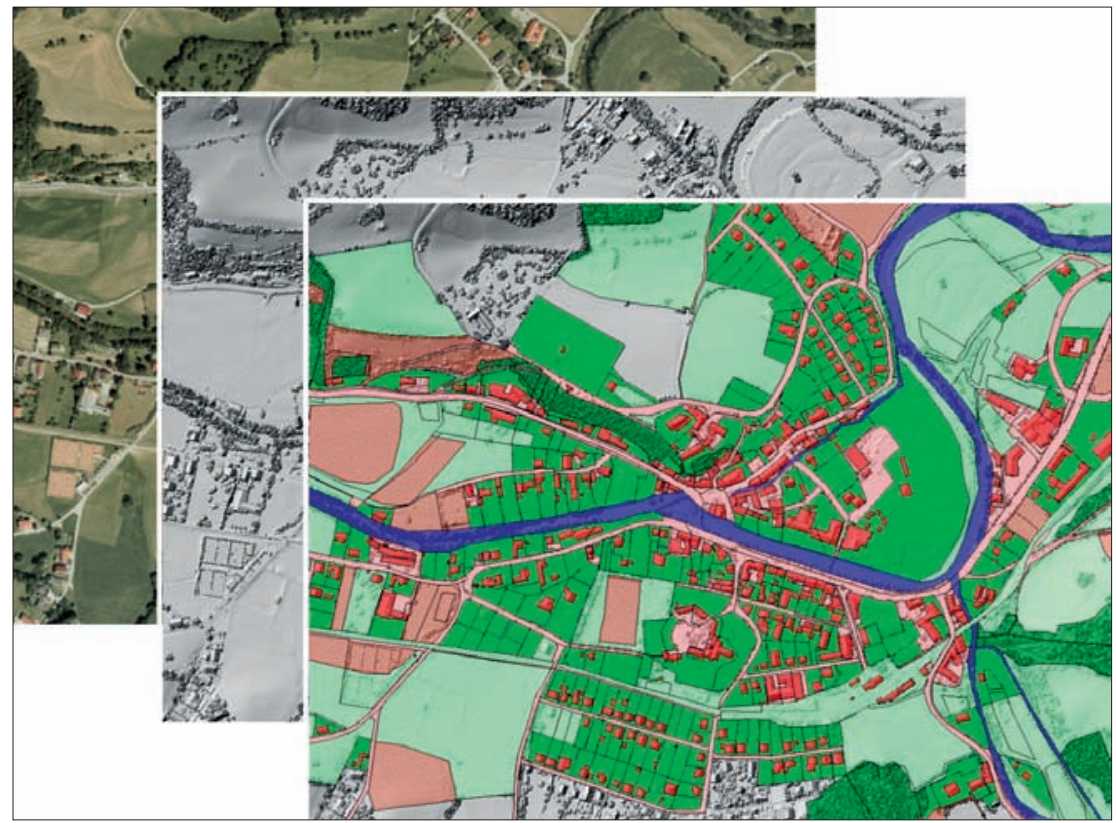

Abb. 8: Erfassung und Interpretation der Flächennutzung.

mobiler Schutzeinrichtungen, das Schließen von Hochwasserverschlüssen etc., welche das Abflussgeschehen beeinflussen.

\subsection{Beobachtete Ereignisse}

Für die Kalibrierung eines Modells sind Kenntnisse der Auswirkungen abgelaufe- ner Hochwasserereignisse von großer Bedeutung. Diese sind häufig in Foto- und Videoarchiven von Gemeinden, Wasserverbänden, Feuerwehren oder Privatpersonen dokumentiert, fallweise stehen detaillierte Hochwasserdokumentationen mit nachgemessenen Hochwassermarken zur Verfügung.

\section{Hydraulische Berechnung}

Im Folgenden soll ein kurzer Überblick über die erforderlichen Bearbeitungsschritte zur Erstellung eines einsatzfähigen hydraulischen Modells gegeben werden. Die angegebenen Schritte gelten im Wesentlichen sowohl für 1d- als auch für 2d-Modelle. Bei den dargestellten Bearbeitungsschritten handelt es sich nicht um eine streng chronologische Abfolge von Tätigkeiten. Der Weg von der Modellerstellung bis zur Präsentation eines guten Ergebnisses ist vielmehr ein iterativer Prozess, welcher laufende Analysen, Prüfungen und Überarbeitungen zur Detaillierung und Verbesserung des Modells erfordert.

\subsection{Modellerstellung}

Die Erstellung des numerischen Modells erfolgt durch Zusammenführung der erwähnten Datengrundlagen in der Regel mittels entsprechender Software. Hierfür wird das Modellgebiet mit einem geeigneten hydraulischen Netz überzogen, die geometrischen und hydraulischen Parameter werden darauf übertragen, die hydraulischen Randbedingungen, in der Regel Zuflüsse an den Zustromrändern und Wasserspiegelhöhen an den Abstromrän- 


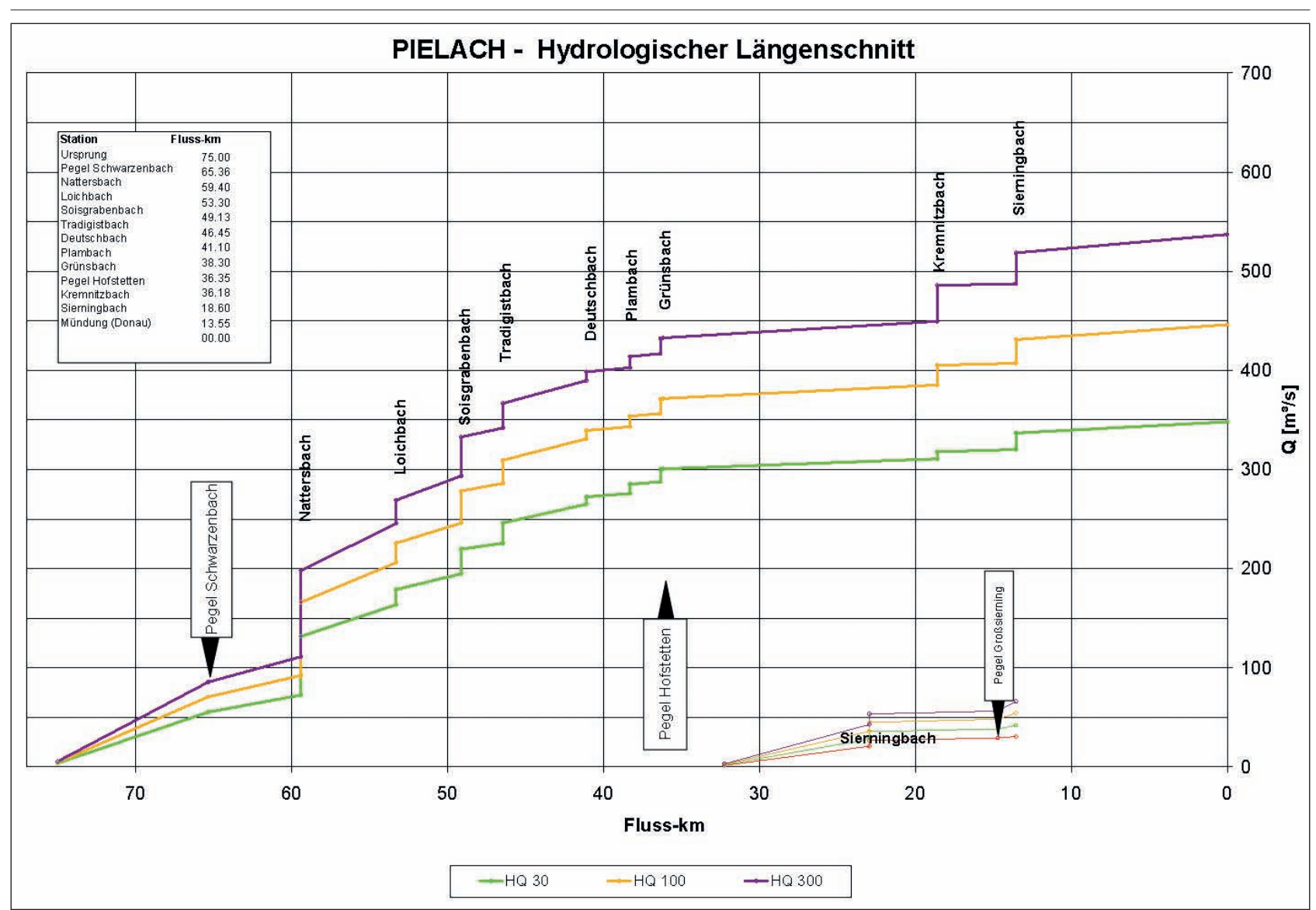

Abb. 9: Hydrologischer Längenschnitt (Beispiel Pielach, NÖ).

dern sowie die weiteren zur Berechnung erforderlichen numerischen Parameter gesetzt. Hierauf können erste Rechenläufe zur Kalibrierung des Modells durchgeführt werden.

Für die meisten der erwähnten Bearbeitungen stehen heute recht ausgereifte Softwareprodukte zur Verfügung, mithilfe derer die Übernahme der Eingangsdaten, deren Aufbereitung und $\mathrm{Pa}$ rametrisierung, die eigentliche Modellrechnung und die anschließende Ausgabe der Berechnungsergebnisse beispielsweise in GIS-kompatiblem Format schrittweise und komfortabel erfolgen kann.

\subsection{Kalibrierung Und Validierung}

In diesem wichtigen Bearbeitungsschritt wird durch Vergleich der Auswirkungen berechneter mit beobachteten Hochwasserereignissen die ausreichende Übereinstimmung des numerischen Modells mit der Natur überprüft. Größere Abweichungen der errechneten von den beobachteten Werten sind durch Modifikationen der Parameter zu korrigieren oder aber zu begründen.

Wichtig bei der Interpretation even- tueller Abweichungen ist die Kenntnis von Unschärfen und besonderen Umständen während des beobachteten Vergleichsereignisses. Diese können sein:

- Unsicherheiten bei Pegellesungen und der Bestimmung der tatsächlich aufgetretenen Abflussmengen

- Unsicherheiten hinsichtlich der genauen Geometrie des Gewässers zum Zeitpunkt des Hochwassers. Später durchgeführte Sohlvermessungen geben unter Umständen nicht das Gewässerbett während des Durchganges der Hochwasserwelle wieder.

- Versagen von Schutzbauten wie Dämmen und Mauern, Ausfall maschineller Einrichtungen (z.B. Pumpwerke zur Polderentwässerung)

- Tatsächliche Ursache lokaler Überflutungen (Aufstau infolge Böschungsbruch, Verklausen von Brücken, aufsteigendes Grund- oder Kanalwasser, ...)

- Mobile Hochwasserschutzmaßnahmen und Notmaßnahmen wie Sandsackwälle

- Abweichen von Vorschriften (Wehrbetriebsordnungen, Offenlassen von Hochwassertoren, ...)
Eine weitere Ursache für Abweichungen kann sein, dass ein in der Natur aufgetretenes Phänomen aufgrund der Art des eingesetzten Modells nicht oder nur unzureichend nachgebildet werden kann, beispielsweise ein Überborden infolge Querneigung des Wasserspiegels in einem 1d-Modell.

Im Idealfall stehen ein gut dokumentiertes Hochwasser zur Kalibrierung und ein weiteres Hochwasser zur Validierung des Modells zur Verfügung. Dabe können jedoch auch zwei mengenmäßig ähnliche Ereignisse infolge anderer Rahmenbedingungen (Bettgeometrie, jahreszeitlich anderer Bewuchs, besondere Vorkommnisse) in der Natur durchaus unterschiedliche Auswirkungen haben.

Steht kein real beobachtetes Ereignis zur Verfügung, so sind für die Plausibilitätsprüfung der Ergebnisse der Sachverstand und die Erfahrung des Bearbeiters gefordert.

\subsection{Berechnung}

Liegt ein plausibles, kalibriertes und im Idealfall an einem weiteren Ereignis validiertes Modell vor, können darauf aufbau- 

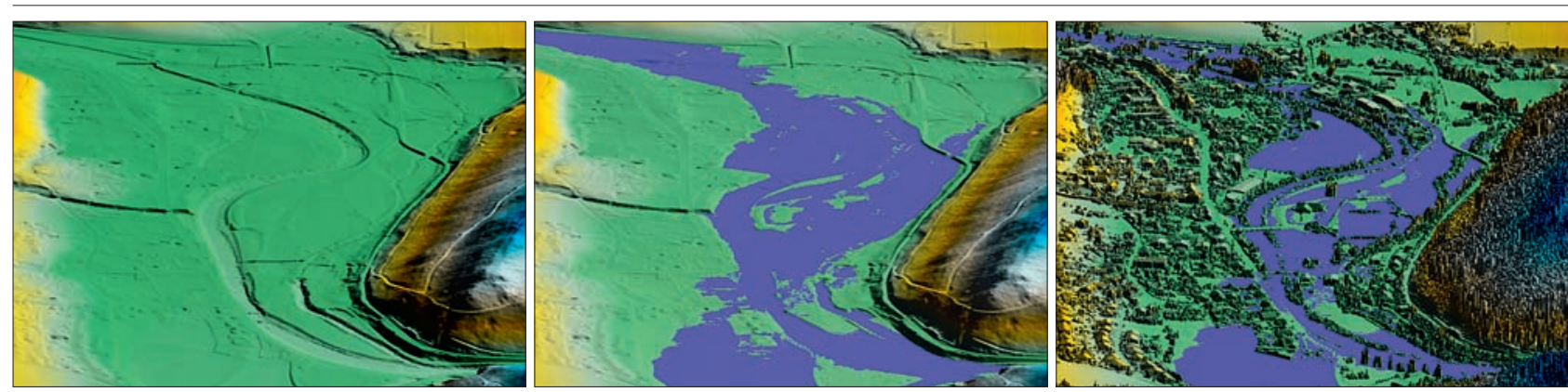

Abb. 10: „Trockenes“ Geländemodell (links), Überflutungssituation bei HQ100 (mitte) und die Visualisierung anhand des Oberflächenmodells (rechts).

end die Bearbeitungen der eigentlichen Fragestellungen durchgeführt werden. Hierfür werden die für die jeweiligen Berechnungsszenarien anzusetzenden hydraulischen Randbedingungen gesetzt und die gegebenenfalls zu untersuchenden geometrischen Veränderungen wie Bettaufweitungen, Vorlandabsenkungen, Schutzdammbauten etc. eingearbeitet.

Nach Durchführung der Berechnung, im Zuge derer die Differenzialgleichungen je nach angewandtem Verfahren gelöst werden, liegen die Abflussverhältnisse in numerischer Form vor und müssen in der Regel vor der weiteren Interpretation und Auswertung visualisiert werden.

\subsection{Visualisierung}

Sie ermöglicht die weitergehende Analyse und Interpretation der Ergebnisse. Für die ansprechende Darstellung von Modellergebnissen als Karten, Schnitte, Ganglinien etc. stehen heute meist integrierte Computerprogramme zur Verfügung. Zudem verfügen die meisten Programme über Datenschnittstellen, mithilfe derer die Berechnungsergebnisse in für GIS oder CADSysteme weiterbearbeitbaren Formaten ausgegeben werden können.

Es lassen sich verständliche Präsentationsunterlagen zur Darstellung von Überflutungsflächen, der Wirkung von Schutzmaßnahmen etc. erstellen, welche im Zuge immer wichtiger werdender Öffentlichkeitsarbeit ein besseres Verständnis komplexer Strömungsvorgänge auch für Laien erlaubt (Abb. 10).

\subsection{Ergebnisprüfung}

Zuvor sind diese Ergebnisse jedoch eingehend zu prüfen, da die Konsequenzen der Ergebnisse in der Regel beträchtlich sind. Die derzeit in mehreren Bundesländern durchgeführten Abflussuntersuchungen mit der Ausweisung der aktuellen HQ30-, HQ100- und HQ300-Überflutungsflächen ziehen beispielsweise häufig Änderungen von Flächenwidmungen wie zum Beispiel Rückwidmungen von Bauland nach sich, Nutzungseinschränkungen und Bauverbote müssen ausgesprochen und andere Zwangsmaßnahmen verhängt werden. Vermittelt auf der anderen Seite ein vielleicht nicht sorgfältig genug geprüftes Ergebnis gar eine falsche Sicherheit vor Hochwassergefahren, so kann dies zu verhängnisvollen und schwer rückgängig machbaren Fehlern in der Raumordnung führen. Und nicht zuletzt bedarf die zielgerichtete Verteilung der ohnedies knapp bemessenen finanziellen Ressourcen zum Schutz vor Hochwässern einer gut abgesicherten Kenntnis der Abflusssituation.

\section{Ausblick}

Seit einigen Jahren sind rasante Entwicklungen der verschiedenen an der hydrodynamischen Modellierung von Oberflächengewässern beteiligten Disziplinen im Gange. Die dabei gemachten Fortschritte betreffen die Grundlagenforschung wie Fernerkundung, Hydrologie und Strömungsmechanik einerseits, die Rechnerleistung sowie die Leistungsfähigkeit und den Komfort der eingesetzten Software andererseits. Die steigende Leistungsfähigkeit von Rechnern in Verbindung mit der entsprechenden Software hat eine Vielzahl interessanter Aspekte. Stichwortartig seien hier einige Punkte ohne Anspruch auf Vollständigkeit angeführt:

- Hydrodynamische 2d-Berechnungen auch großer Flusssysteme können schneller als in Echtzeit durchgeführt werden, wodurch deren Einbindung in Prognose- und Alarmmodelle interessant wird.

- Bereits heute ist es möglich, in vertret- barer Zeit hydrodynamische 2d-Modellierungen von Überflutungsflächen mehrerer Quadratkilometer Größe im unausgedünnten $1 \mathrm{~m}$-Raster des digitalen Geländemodells mit der damit verbundenen hohen Detailauflösung durchzuführen.

- Kopplungen von Abflussmodellen mit Niederschlags-, Sediment- und Schadstofftransport-, Grundwasser- oder Habitatmodellen werden zunehmend interessanter.

- Komplexe kleinräumige, zweidimensional nicht oder nur unzureichend beschreibbare Strömungsvorgänge könnten in verstärktem Ausmaß mittels numerisch um ein Vielfaches anspruchsvolleren 3D-Berechnungen simuliert werden.

Verbunden mit immer komplexer werdenden Modellen sind aber auch gewisse Gefahren. Einerseits können durch die in ihrer Anwendung laufend einfacher werdenden Bearbeitungswerkzeuge die doch recht komplexen Hintergründe, die im Modell durchgeführten Vereinfachungen und die Zusammenhänge einzelner Parameter aus den Augen verloren werden, andererseits besteht die Gefahr, dass durch den intensiven Einsatz numerischer Methoden und anschaulicher Darstellungen am Bildschirm der Planungsraum nur noch als virtuelle Realität wahrgenommen wird. Man muss sich aber stets der Konsequenzen bewusst sein, welche die Ergebnisse derartiger Bearbeitungen in der realen Welt nach sich ziehen. 